\title{
Nanoscale Agent Based Modelling for Nanostructure Development of Cement
}

\author{
E. Cerro-Prada, M.J. Vázquez-Gallo, J. Alonso-Trigueros and A.L. Romera- \\ Zarza
}

E. U. I. T. de Obras Públicas, Universidad Politécnica de Madrid, 28014, Madrid, Spain

\begin{abstract}
Most of macroscopic properties of materials are consequences of processes taking place at the nanoscale. Investigation of phenomena at the sub-micro level to improve the performance of construction materials is one of the main applications of Nanotechnology in Construction. The modelling and simulation of nanostructures is then essential to provide a better understanding of the behaviour of construction materials. We present in this paper an agent-based modelling approach in which a set of interacting agents, capable of organizing themselves dynamically and of adapting to the environment, encapsulate the behaviour of the whole system. This new approach is used for studying the nanostructure development of cement, considering that each individual particle develops its own C-S-H shell critical thickness which triggers the agent for shifting the algorithm to next stage. The algorithm results were compared and found in good agreement with reported experimental work.
\end{abstract}

\section{Modelling the Hydration Process of Cement}

As computational capabilities have evolved rapidly over the last ten years, the ability of mathematical modelling and computation to contribute to the field of materials science has been clearly demonstrated. For porous materials such as hardened cement paste and concrete, major emphasis has been placed on the topic of this work, mathematical and computational modelling of the nanostructure and its potential for predicting mechanical properties and durability. The degree of hydration is one of the key points when studying properties of cement-based materials. For engineering practical purposes, it is very useful to provide calculation procedures or macro models that help to predict mechanical properties as stress and strength, in hardening concrete systems. A computational simulation can be used in producing the hydration curve in terms of initial parameters, as chemical composition of cement, particle size distribution or water/cement ratio.

Computational modelling of the hydration process of cement is found to be a difficult issue due to the large number of factors that should be considered within a three-dimensional spatial domain. Based on our previous work [1], we present in 
this paper an agent-based modelling approach in which a set of agents encapsulate the behaviour of the whole system while its hydration process. This computational modelling technique has been used for studying microstructural models of cement system at two scales: hydrated cement pasted at the micrometer level and calcium silicate hydrated gel at the nanolevel.

\subsection{Agents Encapsulating the Behavior}

In order to construct models involving multiple types of particles and different timing scales, one may choose between two different classes of models: equationbased approaches that evaluate or integrate sets of equations relating the system variables and, in the other hand, agent-based modelling which consists of a set of agents reflecting the behaviour of the various individuals that makes up the behavior of the whole system. In this paper, the latter approach has been adopted. Therefore, a meta-algorithm is developed as a framework for integrating heterogeneous units -agents- to form a complex model, as the cement system is indeed. With this style of scheme integration, the whole system is divided into subsystems, which means that a degree of modularity can be assumed for each subsystem simulated by using a particular algorithm. If this requirement is satisfied, it can be expected an established and well-studied model to be useful when simulating the hydration process of cement.

In the work presented here, this new modelling approach is employed for studying the nanostructure development of cement. A combination of stochastic, deterministic and adaptive rules allows the cement system to evolve from an anhydrous separated state to a bond state with enhanced mechanical properties. The model simulates the two main chemical reactions producing $\mathrm{C}-\mathrm{H}-\mathrm{S}$ gel and portlandite, considering the related anhydrous cement components, $\mathrm{C}_{3} \mathrm{~S}$ and $\mathrm{C}_{2} \mathrm{~S}$, as two autonomous agents, while water and hydration time are viewed as activator agents.

Initially, each agent chooses, at random, one of the two types of particles: type A for $\mathrm{C}_{3} \mathrm{~S}$, or type $\mathrm{B}$ for $\mathrm{C}_{2} \mathrm{~S}$. During the simulation, each agent can take one of the following actions: (i) keep inactive, (ii) react with water, or (iii) stop to reacting with water. The rules for keeping chemically inactive are deterministic as they depend on the type of particle along with the time lasted. Reacting with water is also deterministic by the amount of water available. During the induction period, there is a physical diffusion barrier formed by the reaction products and the hydration process speeds down. The acceleration period follows and hydration process continues faster, until products layer for each particle reaches the critical thickness, according to van Breugel's work [2,3]. When that fact occurs, the deceleration period controls the hydration process at moderate rate. The overall agentbased model algorithm takes the form outlined in Fig. 1.1, left. 

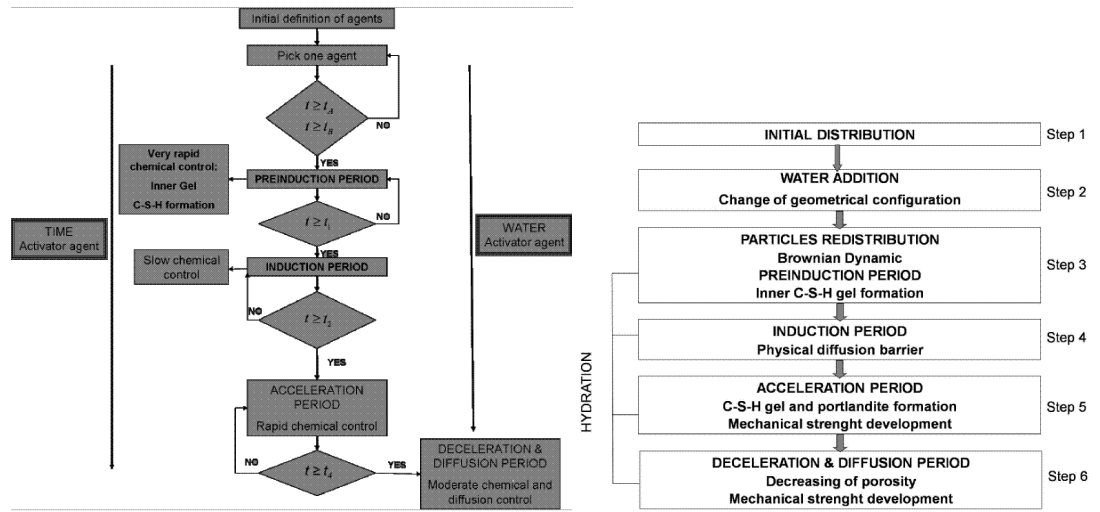

Fig.1.1 Left: Flowchart representation of the agent-based model. Right: Algorithm Flowchart

\subsection{C-S-H Critical Thickness}

The principal aim of this work is to measure the degree of hydration as well as reproduce the nanostructure of the $\mathrm{C}-\mathrm{S}-\mathrm{H}$ phase, resulting from hydration of alite and belite in ordinary Portland cement, through a self-controlled algorithm following the agent-based model described in section 1.1. The algorithm comprises a set of rules based on a systematic ab-initio study of hydration process of Portland cement. The main steps of the algorithm reproduce five hydration stages: preinduction, induction, acceleration, deceleration and diffusion [4].

The hydration kinetics are based on Van Breugel's model [2,3], consisting of an initial phase-boundary reaction in which a layer of C-S-H gel is formed over the surfaces of cement particles followed by a diffusion process, starting at a certain critical time when the product layer around each cement particle reaches the so called transition thickness. The novelty presented here with respect to previous works, has been when considering different rate factors for each individual particle. When hydration occurs, the algorithm randomly assigns to each cement particle its rhythm for decreasing, being this rhythm into a specific time interval that depends on the size and composition of the particle, coherently built from experimental data. Another new ingredient is how the transition to the diffusion period has been modelled. The critical thickness for each particle depends on its size and its chemical type. The values given by the algorithm have shown to be in good agreement with experimental work [2].

Regarding the kinetics of diffusion period, while most previous models presenting the exponential rule for governing the process simplifies the exponential law to a lineal rate, in our model the exponential character on the kinetic law has been preserved. These new approaches could be seen as an indirect but simple way of 
reflecting interactions between particles which, in general, appear to be difficult to simulate.

\section{Experimental Work}

In this study, the material representation is based on the Stroeven's work [5], which simulates the granular structure of cement at the microlevel as a partial distribution of spherical particles (anhydrous cement) diluted in an aqueous matrix water-. This paper presents a non-investigated system configuration based on a two-dimensional granular fluid. Initially, anhydrous cement particles are randomly located throughout a thin film with rectangular base and very small height. When water is added the spatial restriction changes to a cylinder-shaped thin film whose thickness coincides with the previous height. The cement particles are not allowed to overlap any previously placed particle. Immediately after water addition, the hydration process starts and the algorithm simulates the corresponding preinduction, induction, acceleration, deceleration and diffusion periods, as it is shown in Fig. 1.1, right.

The implementation of the algorithm has been performed by using the algebraic computation software Maple [6].The degree of hydration and the thickness of C$\mathrm{H}-\mathrm{S}$ gel both can be obtained in terms of the initial size and proportion of the anhydrous particles, as well as the relative consumption of water, that can be written as a function of the water/cement ratio.

Particle size distribution of the cement is one of the factors which determine the progress of the hydration process. This distribution has been often described in terms of the Rosin-Rammler function [2]. Here, we consider just two chemical types of anhydrous particles: $\mathrm{C}_{3} \mathrm{~S}$, and $\mathrm{C}_{2} \mathrm{~S}$, corresponding to agent-A and agent- $\mathrm{B}$ respectively. Spheres have been chosen for modelling the particle shape (as broadly chosen by most of models, see [2] for a discussion about the suitability of this choice). Regarding size particle, the radius for type A and type B spheres are also set as initial parameters. The water/cement ratio, while most of previous studies consider a set of discrete possible values for this ratio, this algorithm allows this ratio to be an independent variable in terms of which the relative consumption of water can be written. For the rate factor controlling the decreasing radius of cement particles, each one of them has its own rate, taken at random within some interval according to the size and type of particle considered. The exponential character of diffusion period kinetic used here, allows each particle to have a randomly assigned exponent between 1 and 2, in agreement with experimental knowledge.

According to van Breugel's work [2], once the lost cement volume in some interval of time is computed, the volume of $\mathrm{C}-\mathrm{H}-\mathrm{S}$ produced is proportional to that volume by a factor $v_{1}=2.2$, being temperature constant at $20^{\circ} \mathrm{C}$. In the same way, the lost water volume is proportional to the lost cement volume, by a factor equal 
to $v_{2}=0.4$ times the density of cement. The values of these two constants are given by experimental works. Other elements involved in the hydration process of cement, like temperature and different curing conditions, have not been taken into account in this modelling approach.

\section{Results and Discussion}

The algorithm effectiveness has been quantified by building a fineness function to measure the degree of hydration, defined as the ratio of reacted cement volume to initial cement volume, and the relative consumption of water, defined as the ratio of lost water volume to initial water volume, at certain stages of the process. In Table 3.1., the degree of hydration for two different particle sizes, is shown for three different periods of the hydration process. Interpolating these data, it is possible to plot the degree of hydration versus time in these two cases -as it is shown in Fig. 3.1.A- and discuss whether the curves obtained are in good agreement with experimental curves [2]. In Table 3.2., the relative water consumption is shown as a function of the water/cement ratio, being this inversely proportional to the w/c ratio, $\omega$.

\begin{tabular}{|c|c|c|c|}
\hline Hydration Stage & 20 min Preinduction & 28 hours Acceleration & 7 days Deceleration \\
\hline $\begin{array}{c}\mathrm{a}=\text { Degree of hydration } \\
\mathrm{r}_{\mathrm{A}}=10, \mathrm{r}_{\mathrm{B}}=7\end{array}$ & 0.39 & 30.62 & 75.93 \\
\hline $\begin{array}{c}\mathrm{a}=\text { Degree of hydration } \\
\mathrm{r}_{\mathrm{A}}=8, \mathrm{r}_{\mathrm{B}}=6\end{array}$ & 0.29 & 23.32 & 61.96 \\
\hline
\end{tabular}

Table 3.1. Degree of hydration. Ratio between volume of reacted cement to initial cement volume.

A

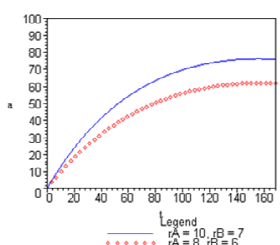

B

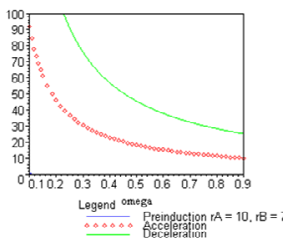

C

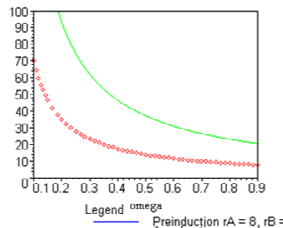

Figure 3.1. A: Degree of hydration, B,C: Relative water consumption

Plotting the relative water consumption versus the water/cement ratio can be done directly from the functions provided by the algorithm, as it is shown in Fig. 3.1, B and C. After the preinduction period, the volume of lost water is so small that the corresponding curve it is not appreciated in the plot. 


\begin{tabular}{|l|c|c|c|}
\hline Hydration stage & 20 min Preinduction & 28 horas Acceleration & 7 days Deceleration \\
\hline $\begin{array}{l}\text { Relative consump. water } \\
\mathrm{r}_{\mathrm{A}}=10, \mathrm{r}_{\mathrm{B}}=7\end{array}$ & $0.027 / \omega$ & $9.188 / \omega$ & $22.778 / \omega$ \\
\hline $\begin{array}{l}\text { Relative consump. water } \\
\mathrm{r}_{\mathrm{A}}=8, \mathrm{r}_{\mathrm{B}}=6\end{array}$ & $0.019 / \omega$ & $6.995 / \omega$ & $18.587 / \omega$ \\
\hline
\end{tabular}

Table 4.2. Relative water consumption

\section{Conclusions}

This work demonstrates that the agent-based molecular formation algorithm for simulation of cement Portland hydration process at the nanoscale, is compatible with the current understanding of the physical-chemical behaviour of the cement grains when they are in contact with water. A great advantage of this computational technique is that the emergent phenomena can be modelled through very simple rules governing the behaviour of each agent as well as the discrete evolution of the system configuration. This computation technique can be helpful for better understanding the correlation between the formation of micro and nano structure as well as the strength development and other mechanical properties of cementitious systems.

\section{References}

[1] E. Cerro-Prada, M.J. Vázquez-Gallo, J. Alonso-Trigueros and A.L. Romera-Zarza. Modelling Hydration Process of Cement Nanoparticles by using an Agent-Based Molecular Formation Algorithm. Proceedings of IASTED International Conference on Nanotechnology and Applications (NANA 2008) September 29 - October 1, 2008, Crete, Greece, 66-71.

[2] K. Van Breugel. Simulation of hydration and formation of structure in hardening cementbased materials, Ph.D. Thesis, Delft University of Tecnology,Delft, The Netherlands, 1991.

[3] K. Van Breugel. Numerical simulation of hydration and microstrutural development in hardening cement-based materials, Cement and Concrete Research, 25, 1995, 319-331.

[4] Kondo et al, $5^{\text {th }}$ ICCC, Tokyo, 1968, Vol II, 203-248.

[5] M. Stroeven \& P. Stroeven, SPACE system for simulation of aggregated matter application to cement hydration, Cement and Concrete Research, 29, 1999, 1299-1304.

[6] Maple 7. 2001 Edition. Copyright@Maplesoft. Waterloo Maple Inc. 